\title{
Biotechnological Studies Concerning the Vegetable Wastes Hydrolyzed Production and Their Use for Yeasts Biomass Development
}

\author{
Ana Despina Ionescu*, Angela Căşărică, Mariana Gratiela Vladu, and Roxana Mădălina Stoica \\ National Chemical-Pharmaceutical for Research and Development Institute-ICCF-Bucharest, Vitan Avenue, Bucharest, Romania \\ *Corresponding Author: Ana Despina Ionescu, National Chemical-Pharmaceutical for Research and Development Institute-ICCF- \\ Bucharest, Vitan Avenue, Bucharest, Romania.
}

Received: October 21, 2019; Published: November 07, 2019

DOI: $10.31080 /$ ASMI.2019.02.0427

\begin{abstract}
This paper presents the laboratory scale obtained results concerning different vegetable wastes hydrolyzed, which means the optimal culture media composition and activity's parameters, in order to obtain a proper level of glucose, able to assure a yeasts culture growth. This paper presents the results obtained by using the new selected yeasts strains but the comparison with the other ones (already identified) allowed us to found that the new isolated strains belong to the species Candida robusta and Saccharomyces cerevisiae.
\end{abstract}

Keywords: Hydrolyzed Wood; Yeasts Biomass Development

\section{Abbreviations}

D1- D24: Indicators for the New Yeast Strains, Isolated from Dried Beech Wood Chips and Verified on Culture Media with Hydrolyzed Wood Wastes, Before Their Identification; TI 0- TI 10: Indicators for the New Yeast Strains, Isolated from Wet Beech Wood Chips and Verified on Culture Media with Hydrolyzed Wood Wastes, Before Their Identification.

\section{Introduction}

A set of biochemical and microbiological analyses was established in order to select some yeast strains presenting a high potential of development on different vegetable wastes, which can improve their use as fodder yeasts, or component of different biological active nutritional supplements enriched with natural vitamins and minerals [1] used for human treatments or as support for Ethanol production [2]. According to the first indicated reference, high level valorization of brewer's yeast (Saccharomyces cerevisiae) together with malt juice, and milled forest fruits (rose hips, blueberry, bilberry, sea berry) can conduct to biological active nutritional supplements enriched with natural vitamins and minerals.
There are some other studies carried out in order to obtain glucose starting from vegetable wastes hydrolyzed [3-11] but each geographical area must verify its own wood sources and moreover, the naturally adapted microbial strains, which can be better used by establishing the optimal parameters and the potential inhibitors of the bioprocess.

The best results were obtained on culture media with 35\% vegetable wastes hydrolyzed, sugar beet molasses, mineral supplements and a pH correction at 6.0. Three selected yeast strains were used on a fermentation media and the results concerning the the yeasts development were verified by optical density (determination meaning the cells number which are present in a well determined liquid volume).

Our conclusion indicates that different types of hydrolyzed vegetable wastes can be recycled by microbiological ways, by using microorganisms selected from natural sources, so that by so called "Green technologies" which seems to act friendly with our environment. 
Biotechnological Studies Concerning the Vegetable Wastes Hydrolyzed Production and Their Use for Yeasts Biomass Development

\section{Materials and Methods}

The samples used as researches' substrate were taken from the paper and cellulose industry's wastes and they were represented by dried beech wood chips, wet beech wood chips and wet and fine beech wood chips, containing 24-25 g/L reducing sugar all of them.

The screening was started on sterilized distilled water (with different rates of dilution) and then on a solid media containing hydrolyzed wood wastes. The yeast strains presenting a better development were then tested on more variants of fermentation media.

The results obtained with these yeasts strains selected from different natural sources were compared with those obtained by using identified yeasts strains kept in the microbial collection of our institute, such as Candida robusta, Candida tropicalis, Candida scotii, Candida arborea, Hansenula sp., Saccharomyces karlsbergensis, Saccharomyces cerevisiae, Saccharomyces diastaticus.

\section{Results and Discussions}

The yeasts strains isolated from those different natural sources were marked at the beginning only with indicators, such as D1D24, T1-T8, etc. depending on their harvest area and their activity was compared with that of different yeasts strains previously identified and included in our institute's microbial collection.

By using this method and some other characters, our selected yeasts strains could belong mostly to the species Candida robusta and Saccharomyces cerevisiae.

\section{Conclusions}

- The main purpose of our work was the valorization of the paper and cellulose industry's wastes by using them as substrate for the yeasts strains development.

- The yeasts strains were isolated from different types of natural sources and the best ones were found by a screening on culture media containing hydrolyzed wood and by comparing them with the efficiency of other identified yeasts existing at our institute's collection.

- This paper presents the results obtained by using the new selected yeasts strains but the comparison with the other ones (already identified) allowed us to found that the new isolated strains belong to the species Candida robusta and Saccharomyces cerevisiae.

\begin{tabular}{|c|c|c|c|c|}
\hline Strain & $24 \mathrm{~h}$ & $48 \mathrm{~h}$ & $72 \mathrm{~h}$ & $90 \mathrm{~h}$ \\
\hline D-21 & +-- & $+\cdots$ & ++++ & ++++ \\
\hline D-22 & +-- & +-- & +-- & ++++ \\
\hline D-4 & +++- & ++++ & ++++ & ++++ \\
\hline D-6 & $\ldots$ & $\ldots$ & +-- & ++- \\
\hline $\mathrm{D}-24 \mathrm{a}$ & $+\cdots$ & ++++ & ++++ & ++++ \\
\hline $\mathrm{D}-24 \mathrm{~b}$ & $+\ldots$ & ++++ & ++++ & ++++ \\
\hline $\mathrm{D}=19 \mathrm{~b}$ & $+\ldots$ & +-- & ++++ & ++++ \\
\hline D-19a & ++-- & ++++ & ++++ & ++++ \\
\hline D-8 & +++- & ++++ & ++++ & ++++ \\
\hline D-20 & +-- & ++++ & ++++ & ++++ \\
\hline D-5 & +++- & ++++ & ++++ & ++++ \\
\hline D-3 & +-- & +++- & ++++ & ++++ \\
\hline D-2 & ++-- & ++++ & ++++ & ++++ \\
\hline D-C 1 & +-- & +-- & +++- & ++++ \\
\hline D-15a & $+\cdots$ & $+\cdots$ & $\ldots$ & - - - \\
\hline D-13a & $\ldots$ & +++- & +++- & +++- \\
\hline TI 0 & $+\cdots$ & ++++ & ++++ & \\
\hline TI 5 & $+\cdots$ & ++++ & ++++ & \\
\hline TI 3 & $+\cdots$ & +-- & +++- & \\
\hline TI 4 & $\ldots$ & +++- & +++- & \\
\hline TI 7 & ++++ & ++++ & ++++ & \\
\hline TI 8 & ++++ & ++++ & ++++ & \\
\hline TI 9 & $\ldots$ & $\ldots$ & +-- & \\
\hline TI 10 & $+\cdots$ & $+\cdots$ & $\cdots$ & \\
\hline D-14 & $-\ldots$ &.-- & $-\ldots$ & \\
\hline D-C3 & $-\ldots$ & $-\ldots$ & $\ldots$ & \\
\hline
\end{tabular}

Table 1: Development of the yeasts strains on solid media containing hydrolyzed wood wastes.

\section{Bibliography}

1. Livia Apostol., et al. "Biologic active food supplements developed upon Saccharomyces cerevisiae usable for food therapy in non-insulin dependent diabetes mellitus". 4th Symposium with International Participation Drug Research between Information and Life Sciences Bucharest (2006).

2. Carlos Martin., et al. "Ethanol production from enzymatic hydrolysates of sugarcane bagasse using recombinant xyloseutilising Saccharomyces cerevisiae". Enzyme and Microbial Technology 31.3 (2002): 274-282. 
3. Yoshizawa K. "Treatment of waste water discharged from a sake brewery using yeast". Hakko Kogaku Zasshi 56.4 (1978): 389-395.

4. Elwin F Harris. "Molasses and yeast from wood".

5. Rocío Gómez-Pastor., et al. "Recent Advances in Yeast Biomass Production". Intech Open (2011).

6. Elliott Douglas C., et al. "Biorefinery Concept Development Based on Wheat Flour Milling". Conference Proceedings: Fuel Chemistry Division Preprints American Chemical Society National Meeting 47.1 (2002): 361-362.

7. Solange Innes Mussatto and Ines Conceicao Roberto. "Alternatives for detoxification of diluted-acid lignocellulosic hydrolyzates for use in fermentative processes: a review". Bioresources Technology 93.1 (2004): 1-10.

8. Anne S Meyer. "Enzyme technology for precision functional food ingredient processes". Annals of The New York Academy of Sciences 1190.1 (2010): 126-132.

9. Renata Baranauskienė and Rita Kazernavičiūtè. "Agrorefinery of Tanacetum vulgare L. into valuable products and evaluation of their antioxidant properties and phytochemical composition". Industrial Crops and Products 60 (2014): 113-122.

10. Aparna S., et al. "Wheat and Its Application in Dairy Products: A Review". Journal of Dairy Science and Technology 4.2 (2015): 19-34.

11. Povilaitis Darius. "Biorefining the by-products of plant material processing into valuable functional ingredients by applying various extraction techniques". Kaunas University of Technology (2016).

\section{Volume 2 Issue 12 December 2019}

(C) All rights are reserved by Ana Despina Ionescu., et al. 